\title{
Rivastigmin verbessert die Gangstabilität
}

Fragestellung: In dieser Arbeit sollte am Universitätsklinikum in Bristol untersucht werden, ob Parkinson-Patienten mit einer Gangunsicherheit durch den Acetylcholinesterase-Hemmstoff Rivastigmin erfolgreich therapiert werden können.

Hintergrund: Stürze sind bei Parkinson-Patienten ab dem Hoehn \& Yahr-Stadium III ein häufiges Problem, das vermutlich mit den bisherigen, uns zur Verfügung stehenden, Medikamenten nicht gebessert werden kann. Prospektive Studien hatten gezeigt, dass bis zu $70 \%$ aller Parkinson-Patienten zumindest einmal im Jahr stürzen und $40 \%$ regelmäßige Stürze berichten. Nach Auftreten gehäufter Stürze ist die mittlere Überlebenszeit nur noch sechs Jahre. Folgen dieser Stürze sind offensichtlich, nämlich nicht nur die Frakturen und Verletzungen, die Angst vor weiteren Stürzen, sondern insbesondere die Krankenhauseinweisung und das Auftreten von Pneumonien und Embolien. Von Seiten des Neurotransmittersystems scheint eine cholinerge Dysfunktion für Freezing und die posturale Instabilität verantwortlich zu sein. Darüber hinaus gibt es Hinweise, dass der pedunculopontine Nucleus für unseren sicheren Gang unabdingbar ist und bei Parkinson-Patienten strukturelle Auffälligkeiten und einen cholinergen Verlust aufweist.

Patienten und Methodik: In die randomisierte, doppelblinde, placebokontrollierte Phase-II-Studie wurden Patienten aufgenommen, die zumindest einen Sturz im Vorjahr auf-

Henderson EJ, Lord SR, Brodie MA et al. Rivastigmine for gait stability in patients with Parkinson's disease (ReSPonD): a randomised, double-blind, placebo-controlled, phase 2 trial Lancet Neurol 2016; 15: 249-58 wiesen, mindestens 18 Meter ohne Hilfe gehen konnten und bislang keinen Acetylcholinesterasehemmer eingenommen hatten. Die Patienten durften keine Demenz aufweisen. Die Randomisie- rung erfolgte 1:1 zwischen Placebo und Verum. Rivastigmin wurde langsam von $3 \mathrm{mg}$ pro Tag bis zur angestrebten Dosis von $12 \mathrm{mg}$ pro Tag im Laufe von zwölf Wochen hochtitriert. Primärer Endpunkt war die Differenz der Schrittzeitvariabilität nach 32 Wochen im Vergleich zum Ausgangswert unter Berücksichtigung von Alter, Kognition, Schrittzeitvariabilität und Anzahl an Stürzen im vorangegangenen Jahr. Die Schrittzeitvariabilität wurde mit einem Accelerometer untersucht, wobei die Patienten während des Gehens Worte, die mit dem gleichen Buchstaben begannen, laut vor sich hersagen mussten. In einem komplizierteren Setting mussten die Patienten während des Gehens Worte aufsagen, die alternativ zwei vorgegebene Buchstaben des Alphabetes als ersten Buchstaben enthalten mussten.

Ergebnisse: Von Oktober 2012 bis Ende März 2013 wurden 130 Patienten randomisiert (65 Rivastigmin, 65 Placebo). Nach 32 Wochen konnten noch 59 Patienten in der Placebo- und 55 in der Rivastigmin-Gruppe verglichen werden. Die Patienten der Rivastigmin-Gruppe zeigten eine signifikante Verbesserung der Schrittzeitvariabilität bei Anwendung des einfachen Worttests. Bei Verwendung des komplexeren Tests wurde kein signifikanter Unterschied zwischen den beiden Gruppen festgestellt.

In der Rivastigmin-Gruppe zeigte sich eine Reduktion der Sturzrate pro Monat um 45\%. Dazu kam ein kleiner, aber doch signifikanter Gewinn bezüglich der Ganggeschwindigkeit. Gastrointestinale Nebenwirkungen waren mit zirka $31 \%$ unter Rivastigmin deutlich häufiger als unter Placebo.

Schlussfolgerungen: Rivastigmin verrbessert die Gangstabilität bei Patienten mit Parkinson bei akzeptabler Tolerabilität und Sicherheit. Die Autoren vermuten, dass das Ansprechen auf Rivastigmin in der Verbesserung der Gangvariabilität, Geschwindigkeit und der Balance beruht. Die Autoren empfehlen die Durchführung einer größeren randomisierten Phase-III-Studie.

\section{- Kommentar von Heinz Reichmann, Dresden}

\section{Eine weitere Studie erscheint notwendig und vielversprechend}

Aus meiner Sicht ist dies eine ernstzunehmende Studie, obwohl keine große Anzahl an Parkinson-Patienten rekrutiert wurde, aber doch eine so ausreichende Zahl an Parkinson-Patienten mit Stürzen in der Vorgeschichte untersucht wurde, dass das Ergebnis neugierig macht. Die zeitliche Assoziation zwischen Stürzen und kognitiver Verschlechterung bei Parkinson-Patienten hat sicherlich indirekt dazu beigetragen, dass viele unserer deutschen Patienten Rivastigmin, der einzige für kognitive Defizite bei Parkinson-Syndrom zugelassene Cholinesterasehemmer, bereits erhalten haben. Es ist somit anekdotisch recht schwierig zu erahnen, ob wir damit indirekt unseren $\mathrm{Pa}$ tienten nicht nur eine verbesserte Kognition, sondern auch eine Sturzprophylaxe haben zukommen lassen. Ich selbst würde somit den Vorschlag der Autoren nachhaltig unterstützen, dass nunmehr eine multizentrische, randomisierte, doppelblinde Studie mit Rivastigmin bei Parkinson-Patienten mit Stürzen oder Sturzgefahr notwendig und vielversprechend erscheint.

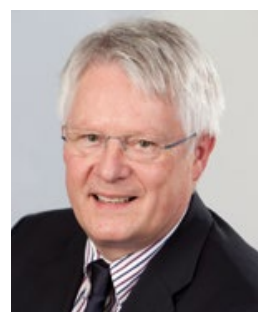

Prof. Dr. med. Heinz Reichmann, Dresden

Direktor der Klinik und Poliklinik für Neurologie, Klinikum Carl Gustav Carus der TU Dresden, Dekan der Medizinischen Fakultät. E-Mail: heinz.reichmann@ uniklinikum-dresden.de 